\title{
Influence of crystalline and shape anisotropy on electrochromic modulation in doped semiconductor nanocrystals
}

\author{
Sungyeon Heo, ${ }^{\dagger \S}$ Shin Hum Cho, ${ }^{\dagger}, "$ Clayton J. Dahlman, ${ }^{\#}$ Ankit Agrawal, ${ }^{\ddagger}$ and Delia J. Milliron ${ }^{*}$, \\ † McKetta Department of Chemical Engineering, The University of Texas at Austin, Austin, Texas 78712, \\ United States \\ ‡ The Molecular Foundry, Lawrence Berkeley National Laboratory, 1 Cyclotron Road, Berkeley, \\ California 94720, United States \\ \# Materials Department, University of California, Santa Barbara, California 93106, United States \\ $\S$ Current affiliation: Department of Electrical Engineering, Princeton University, Princeton, New Jersey \\ 08544, United States \\ I| Current affiliation: Samsung Electronics, Samsung Semiconductor R\&D, Hwaseong, Gyeonggi-do \\ 18448, Republic of Korea
}

*Corresponding author: milliron@che.utexas.edu 


\begin{abstract}
Localized surface plasmon resonance (LSPR) modulation appearing in the near-infrared range in doped semiconductor nanocrystals enriches electrochromic performance. Although crystalline and shape anisotropies influence LSPR spectra, study of their impact on electrochromic modulation are lacking. Here, we study how crystalline anisotropy in hexagonal cesium-doped tungsten oxide nanorods and nanoplatelets affects essential metrics of electrochromic modulation - coloration efficiency (CE) and volumetric capacity - using different sizes of electrolyte cations (tetrabutylammonium, sodium, and lithium) as structurally sensitive electrochemical probes. Nanorod films show higher CE than nanoplatelets in all of electrolytes owing to low effective mass along the crystalline c-axis. When using sodium cations, which diffuse through one-dimensional hexagonal tunnels, electrochemical capacity is significantly greater for platelets than for nanorods. This difference is explained by the hexagonal tunnel sites being more accessible in platelets than in nanorods. Our work sheds light on the role of shape and crystalline anisotropy on charge capacity and $\mathrm{CE}$ both of which contribute to overall modulation.
\end{abstract}

\title{
TOC GRAPHICS
}

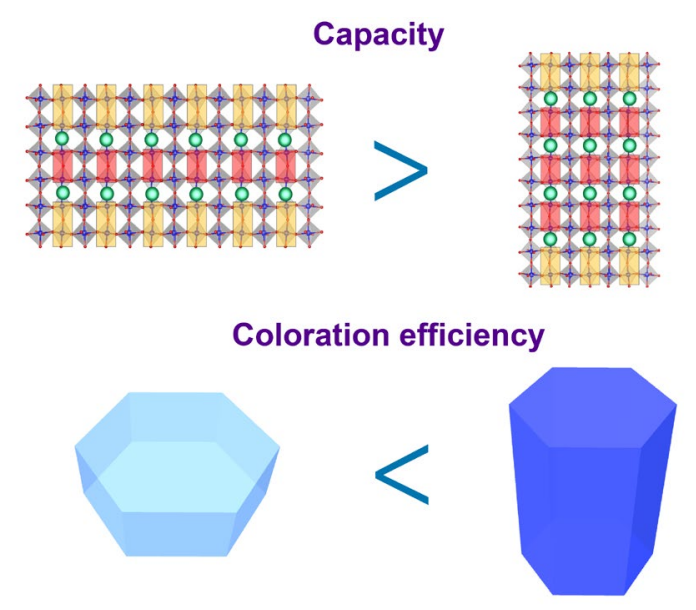




\section{MAIN TEXT}

Doped metal oxide nanocrystals have shown promise as a key component for next generation electrochromic windows. ${ }^{1-5}$ Films of semiconductor nanocrystals can accumulate charge with an electrochemical bias, and scatter light in the visible to near-infrared range by localized surface plasmon resonance (LSPR). Doped metal oxides have low carrier concentrations $\left(10^{18} \sim 10^{22} / \mathrm{cm}^{3}\right)$ compared to intrinsic metals, ${ }^{6}$ so electrochemical charging induces large modulations in optical conductivity and LSPR response. In addition, nanocrystal electrochromic films have short charge diffusion lengths, enabling fast switching, and can be fabricated with low-temperature processing that allows flexible form factors. ${ }^{7}$ Semiconducting nanocrystals that undergo electrochemical ion-insertion, such as $\mathrm{Nb}$-doped $\mathrm{TiO}_{2}$ and $\mathrm{Nb}_{12} \mathrm{O}_{29}$, can even exhibit dual-mode modulation in both the visible and near-infrared range due to distinct charge compensation mechanisms. ${ }^{8-10}$

While the spectral characteristics of electrochromic nanocrystals (visible glare control, solar infrared modulation, and color neutrality) are regularly studied, ${ }^{11-13}$ the practical concern of maximum optical modulation range receives less attention. One well-established metric for the optical modulation is coloration efficiency (CE), defined as the change in optical density $(\triangle \mathrm{OD})$ of the coating per injected charge density $\left(\mathrm{mC} / \mathrm{cm}^{2}\right)$ at a designated wavelength where the charge is normalized by the projected area of active electrochromic material. ${ }^{14}$ The maximum optical modulation a film can achieve is governed by the intrinsic spectral response of the material and thickness of the film. If the intrinsic CE of the material is greater, then the film can be thinner, manufactured with less raw materials, and requires less charge to switch. Similarly, if the material has more volumetric charge capacity, then greater optical modulation can be achieved in thinner coatings. Intrinsic CE and charge capacity are both influenced by the mechanism of charge compensation in electrochromic nanocrystal films, especially in ion-inserting semiconductors such as doped $\mathrm{WO}_{3}, \mathrm{Nb}_{2} \mathrm{O}_{5}$, and $\mathrm{TiO}_{2}$. Capacity and $\mathrm{CE}$ can be influenced by the intracrystalline structure and presence of interstitial or antisite defects. ${ }^{15-16}$

The optical spectra of plasmonic nanocrystals depend significantly on their morphology, though the manifestation of shape effects in the dynamic spectra under electrochemical charging has not been reported. Anisotropy in nanocrystal shape (e.g. rods, platelets, stars) can lead to distinct optical modes in their LSPR, exemplified by the example of transverse and longitudinal modes in noble metal nanorods. ${ }^{17-18}$ Nanocrystal shape also influences the LSPR spectrum of colloidal particles of degenerate metal oxide compositions commonly used for electrochromic coatings, such as doped $\operatorname{In}_{2} \mathrm{O}_{3}$ nanocubes. ${ }^{19}$ The size of plasmonic metal oxide nanocrystals also impacts their LSPR, and in films size has additional influence on optical properties because of surface depletion and inter-nanocrystal LSPR coupling effects, which may also change the magnitude and energy of optical modulation during charging. ${ }^{20-22}$ The distinct LSPR modes in anisotropically shaped plasmonic nanocrystals are expected to impart different intensities and CE during electrochromic switching.

Unlike noble metals, semiconductor oxides can also possess anisotropy due to the underlying crystal structure. The effects of crystalline anisotropy on the electronic properties of nanostructured thermoelectric devices have been studied, ${ }^{23}$ but its impacts on LSPR are only recently gaining attention. Our group revealed that crystalline anisotropy affects optical properties in metal oxide nanocrystals because of the difference in effective mass along each lattice direction. Kim et al. demonstrated that unexpected peak splitting in isoprism (aspect ratio: 1) hexagonal tungsten oxide nanocrystals is a consequence of crystalline anisotropy, and that an interplay of shape and crystalline anisotropy determines plasmonic line 
shape and intensity. ${ }^{24}$ Dahlman et al. invoked crystalline anisotropy to explain the unexpectedly low energy of LSPR in degenerately doped or charged $\mathrm{TiO}_{2}{ }^{25}$

The electrochromic response of plasmonic nanocrystals with both shape and crystalline anisotropy has received little attention, despite the significant influence of these anisotropies on LSPR modulation. LSPR mode along the lowest effective mass lattice direction shows the greatest intensity upon charged or doping in $\mathrm{TiO}_{2}$ nanocrystals. ${ }^{25}$ Similarly, elongation in nanocrystal shape can increase the relative cross section of particular LSPR modes, ${ }^{18}$ and might change intrinsic CE at different wavelengths. The combination of shape and crystalline anisotropy can either magnify or reverse these trends depending on the relationship between the orientation of the low effective mass direction and the elongated direction. Moreover, volumetric charge capacity is also expected to be influenced by crystalline and shape anisotropy if ion insertion favors particular lattice directions. Thus, shape and crystalline anisotropy in doped metal oxide nanocrystals can have significant implications for the practical performance of plasmonic electrochromic devices.

Here, we systematically elucidate the influence of shape and crystalline anisotropies in hexagonal cesium-doped tungsten oxide (h-Cs: $\mathrm{WO}_{3}$ ) nanocrystal films on essential metrics of electrochromic modulation, namely $\mathrm{CE}$ and charge capacity. The interaction between each source of anisotropy is differentiated by engineering the shape of h-Cs: $\mathrm{WO}_{3}$ nanocrystals (nanorods and nanoplatelets) with identical crystal phase. Extrinsic properties of the film, such as thickness and porosity, are controlled between each sample to measure the intrinsic OD and volumetric capacity of each nanocrystal shape. As a result, we reveal that interplay of crystalline and shape anisotropy induces 1) higher intrinsic CE at the dominant LSPR wavelength $(1700 \mathrm{~nm})$ in nanorods than in nanoplatelets, and 2) significantly higher volumetric capacity (and thus optical modulation) in nanoplatelets charged in a $\mathrm{Na}^{+}$electrolyte due to onedimensional diffusion through hexagonal tunnel sites.
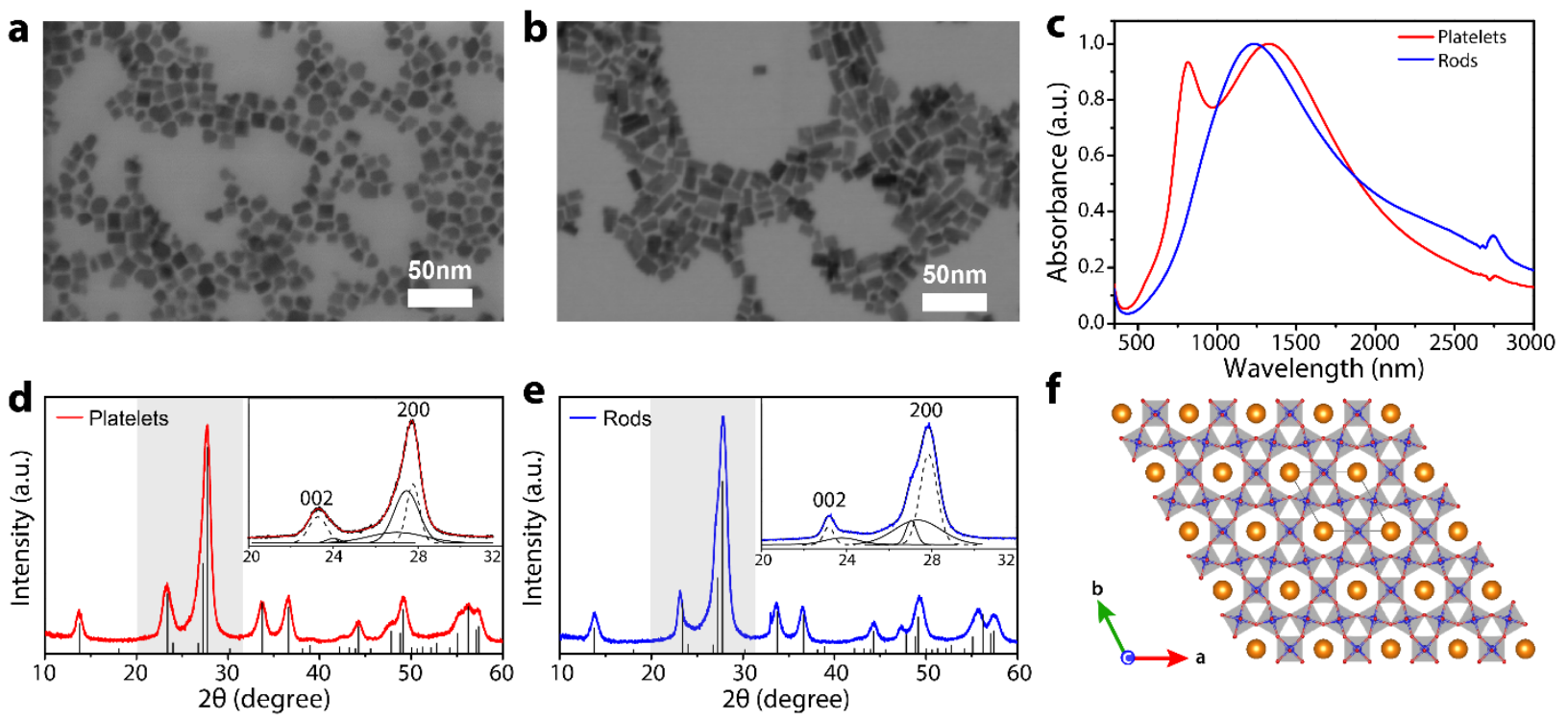

Figure 1. h-Cs: $\mathrm{WO}_{3}$ nanoplatelets and nanorods. $(\mathrm{a}, \mathrm{b})$ Scanning transmission electron microscopy (STEM) images of (a) nanoplatelets and (b) nanorods. (c) UV-visible-NIR spectra of nanoplatelets and nanorods dispersed in tetrachloroethylene. $(\mathrm{d}, \mathrm{e}) \mathrm{XRD}$ patterns for nanoplatelets and nanorods, and corresponding hexagonal $\mathrm{Cs}_{0.22} \mathrm{WO}_{3}$ reference pattern (ICSD \#56223). Insets are the magnified XRD pattern used for size 
and shape analysis using (002) and (200) peaks indicated by dashed lines. (f) Illustration of hexagonal cesium-doped tungsten oxide crystal structure viewed along the [001] direction.

h-Cs: $\mathrm{WO}_{3}$ nanocrystals were synthesized by colloidal methods to vary nanocrystal shape while preserving crystalline phase, surface area, and volume. Nanocrystals of h-Cs: $\mathrm{WO}_{3}$ have been synthesized as hexagonal prisms with shapes tunable between elongated nanorods and flat nanoplatelets. ${ }^{24}$ Surface charging may contribute a large share of volumetric capacity in nanocrystalline tungsten oxide electrodes, ${ }^{26-}$ ${ }^{27}$ so the specific geometric surface area must be controlled to compare intrinsic CE and volumetric capacity. Nanocrystal size can also influence peak shifts and intensity changes under electrochemical charging owing to changes in the degree of LSPR coupling, so nanocrystal volume also needs to be similar to avoid these convoluting effects. ${ }^{20}$ The previously reported synthetic methods were modified to fine-tune nanocrystal size and shape and preserve similar surface area and volume between two samples: nanorods and nanoplatelets. ${ }^{24}$ Thus, the interplay between anisotropy in nanocrystal shape and the intrinsic crystalline structure can be directly studied.

X-ray diffraction (XRD) of deposited films reveals that both nanocrystals share the same crystalline phase (Figure 1d,e), consistent with $\mathrm{Cs}_{0.29} \mathrm{WO}_{3}$ (ICSD \#56223). ${ }^{28}$ Scherrer analysis indicates the dimensions of each nanocrystal sample; nanorods have a mean size of $15.7 \mathrm{~nm}$ (height) $\times 9.0 \mathrm{~nm}$ (width) with an aspect ratio of 1.74, while nanoplatelets have a mean size of $8.5 \mathrm{~nm}$ (height) $\times 12.0 \mathrm{~nm}$ (width) with an aspect ratio of 0.71 (Table 1). Dimensions obtained from image analysis of scanning transmission electron microscopy (STEM) measurements are consistent with XRD results, and provide a measure of size polydispersity (Figure 1a,b and Figure S1). Despite both nanocrystal samples sharing same crystal phase with similar surface area and volume (Table 1), UV-visible-NIR spectra of dispersions are strikingly different. Notably, only the platelets show a distinct peak around $810 \mathrm{~nm}$, separate from the larger, broader peak observed in both samples around $1300 \mathrm{~nm}$. This result is consistent with a previous report that observed a large peak separation $\left(4000 \mathrm{~cm}^{-1}\right)$ between LSPR modes in platelets because of the crystalline anisotropy effect (Figure 1c) ${ }^{24}$ Although detailed comparisons of spectroelectrochemical properties were made between these two specific synthetic products (one batch each of nanorods and nanoplatelets), the synthetic methods were robust in producing structurally similar products and all the trends highlighted below that compare rods to platelets were consistent across several synthesized batches.

$\begin{array}{ccc}\text { XRD Scherrer analysis } & \text { rods } & \text { platelets } \\ \text { width }(\mathrm{nm}) & 9.0 & 12.0 \\ \text { height }(\mathrm{nm}) & 15.7 & 8.5 \\ \text { aspect ratio } & 1.74 & 0.71 \\ \text { surface area }\left(\mathrm{nm}^{2}\right) & 630 & 603 \\ \text { volume }\left(\mathrm{nm}^{3}\right) & 1101 & 1060\end{array}$

Table 1. Dimensions of synthesized nanorods and nanoplatelets estimated from XRD measurements. Surface area and volume were calculated by the equations in Figure S1.

Comparison of spectroelectrochemical properties between different nanocrystal films must be judicious because extrinsic film parameters such as thickness, porosity, and roughness have large influences. To control these parameters, we fabricated optical quality, uniform thin films with similar thickness 
between each nanocrystal sample by spin-coating (Figure S2). Thickness was measured by profilometer, and ellipsometric porosimetry was used to measure porosity (Figure S3). Minor sample-to-sample variation was minimized by fabricating each set of films, for either the nanorod and nanoplatelet samples, from the same synthesis batch. Thus, while slight variations in thickness and porosity are observed between the nanorod and nanoplatelet films, all films of the same nanocrystal batch have same properties. (Porosity and thickness of nanorod films are $17.5 \%$ and $124 \mathrm{~nm}$, while porosity and thickness of nanoplatelet films are $14.5 \%$ and $133 \mathrm{~nm})$.

Total optical modulation a film can achieve is a function of both the differential CE and the total charge capacity at a particular electrochemical potential. Electrochromic devices with high CE and capacity exhibit large optical modulation with less energy, so thinner electrochromic coatings can be fabricated to improve switching rates, transparency, and cost effectiveness. Traditionally, capacity is normalized by the projected film area. ${ }^{29-33}$ As shown in Figure S4a,b, when charge is normalized by projected area only, large charge density is obtained from larger film thickness, thereby introducing greater overall modulation. As such, this traditional approach obfuscates the intrinsic material properties that control practical device characteristics, making it hard to compare materials for their potential utility in electrochromic films. Thus, intrinsic measures of OD and capacity are required to optimize both optical modulation and film thickness of electrochromic coatings.

To study the intrinsic spectroelectrochemical properties of each nanocrystal shape, per unit volume of h-Cs: $\mathrm{WO}_{3}$, we strictly normalized measured OD and charge capacities by the volume of nanocrystals. First, we normalized the OD with respect to the volume of nanocrystals based on the measured volume fraction (from porosity) and film thickness. This approach is a film analog of the extinction coefficient calculated for nanocrystals dispersed in solution, where path length is analogous to film thickness and volume fraction is analogous to particle concentration. ${ }^{34-35}$ It should be noted that projected area of entire nanocrystal film is not considered in our normalization as OD is not proportional to the projected area. We then estimated the volumetric charge capacity by normalizing total charge with respect to the volume fraction, film thickness, and projected area (Figure S4). In this case, projected area is considered because capacity is measured across the entire film, while the optical beam path projects through only a fixed fraction of the film. Details of the calculation are provided in the Supplemental Information. This volume normalization approach enables a direct comparison of the intrinsic OD and volumetric capacity between the nanorod and nanoplatelet films (Figure S4b,c).

The electrochromic responses of nanorod and nanoplatelet films were first measured with a bulky non-inserting electrolyte to isolate the influence of surface charging. Ion insertion by smaller cations such as $\mathrm{Na}^{+}$is highly anisotropic in doped $\mathrm{WO}_{3}$ nanocrystals, ${ }^{26}$ and convolutes the effects of shape and crystalline anisotropy on electrochromic modulation. Thus, we first tested spectroelectrochemical properties with $0.1 \mathrm{M}$ tetrabutylammonium $\left(\mathrm{TBA}^{+}\right)$perchlorate in propylene carbonate as the electrolyte. $\mathrm{TBA}^{+}$is too large to insert into the h-Cs: $\mathrm{WO}_{3}$ lattice, ${ }^{36}$ so only surface charging mechanisms contribute to the capacity. First, we compared the traditional projected area-normalized CE and capacity values, and found a larger charge capacity for the nanoplatelet film than the nanorod film (Figure S5). However, when these values are normalized by nanocrystal volume, taking into account the slightly larger thickness of the platelet film (133 $\mathrm{nm})$ than the nanorod film $(124 \mathrm{~nm})$, the normalized capacity is very similar for each nanocrystal shape (Table 2). The surface-area-to-volume ratio for both nanocrystal shapes are very similar (Table 1), so this result is consistent with a surface charging mechanism. 


\begin{tabular}{cccccccc} 
electrolytes & \multicolumn{3}{c}{ rods } & & \multicolumn{3}{c}{ platelets } \\
\cline { 2 - 4 } & $\begin{array}{c}\mathrm{CE} \\
\left(\mathrm{cm}^{2} / \mathrm{C}\right)\end{array}$ & $\begin{array}{c}\text { capacity } \\
\left(\mathrm{mC} / 10^{17} \mathrm{~nm}^{3}\right)\end{array}$ & $\begin{array}{c}\mathrm{OD}_{\max } \\
\left(10^{-4} \mathrm{~nm}^{-1}\right)\end{array}$ & & $\begin{array}{c}\mathrm{CE} \\
\left(\mathrm{cm}^{2} / \mathrm{C}\right)\end{array}$ & $\begin{array}{c}\text { capacity } \\
\left(\mathrm{mC} / 10^{17} \mathrm{~nm}^{3}\right)\end{array}$ & $\begin{array}{c}\mathrm{OD}_{\max } \\
\left(10^{-4} \mathrm{~nm}^{-1}\right)\end{array}$ \\
\hline $\mathrm{TBA}^{+}$ & 200 & 8.1 & 15.5 & & 150 & 8.2 & 12.3 \\
\hline $\mathrm{Na}^{+}$ & 212 & 19.6 & 36.3 & & 156 & 25.0 & 33.3 \\
\hline $\mathrm{Li}^{+}$ & 137 & 78.3 & 72.9 & & 126 & 78.4 & 62.6
\end{tabular}

Table 2. Intrinsic properties of $\mathrm{CE}$ at $1700 \mathrm{~nm}$, capacity, and maximum OD of nanorod and nanoplatelet film with $\mathrm{TBA}^{+}, \mathrm{Na}^{+}$, and $\mathrm{Li}^{+}$electrolytes. All parameters are obtained based on the volume normalization process.

a

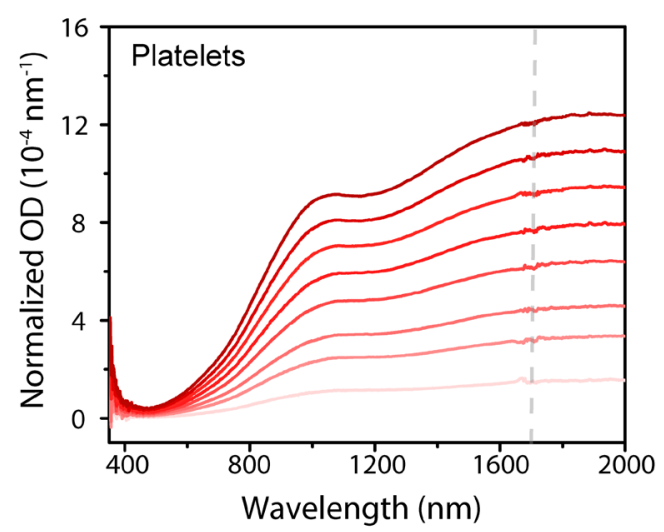

C

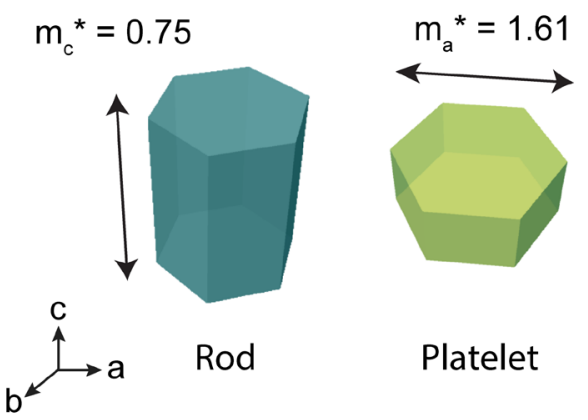

b

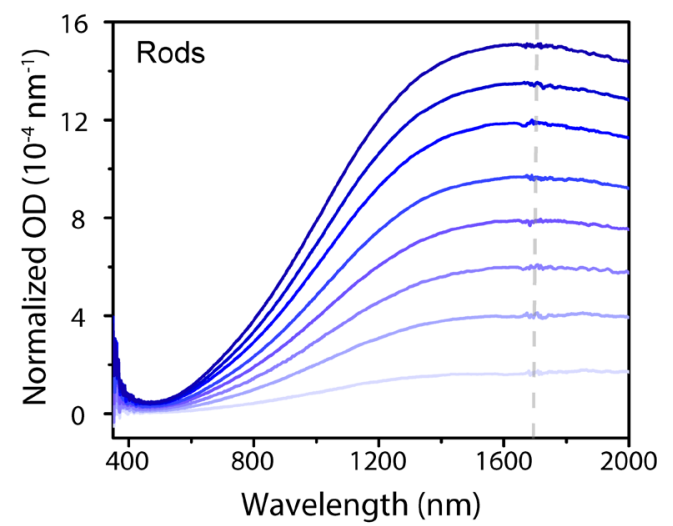

d

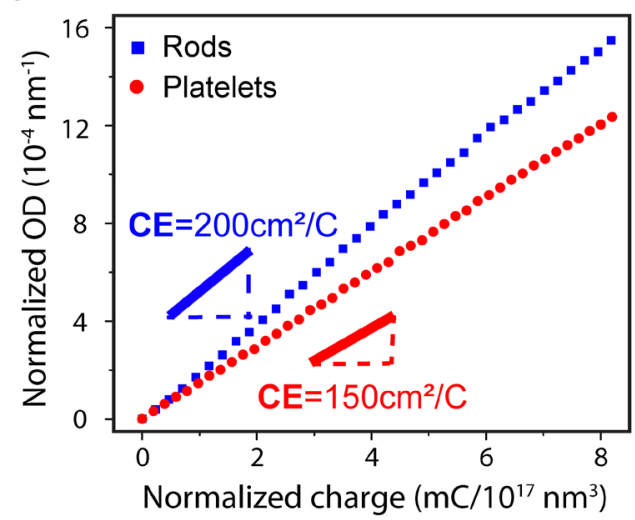

Figure 2. Electrochromic modulation of nanoplatelets and nanorods with $\mathrm{TBA}^{+}$electrolyte. (a,b) Normalized extinction spectra of nanoplatelet and nanorod films. Each incremental graph corresponds to the same increase of normalized charge capacity $\left(1 \mathrm{mC} / 10^{17} \mathrm{~nm}^{3}\right)$. The dashed line lies at the wavelength used for the OD change in (d). (c) Effective mass along different axes in nanorods and nanoplatelets. (d) Normalized OD at $1700 \mathrm{~nm}$ and injected charge in nanoplatelet and nanorod films.

Upon charging in $\mathrm{TBA}^{+}$electrolyte the intrinsic capacity of the two nanocrystal morphologies are the same, but the nanorods have higher intrinsic CE than the nanoplatelets. Figure 2 demonstrates the difference in electrochromic optical modulation between the two films. Similar to optical spectra of NC 
dispersions (Figure 1c), the platelets show two peaks and the rods have only one. Features in the film spectra are red-shifted and much broader because of LSPR coupling between nanocrystals. ${ }^{20}$ However, the maximum intrinsic coloration achieved near the LSPR peak $(1700 \mathrm{~nm})$ is greater for nanorods than nanoplatelets (Figure 2a,b). Intrinsic CE values obtained from the slopes of the curves (Figure 2d) corroborate this result: the nanorods (intrinsic $\mathrm{CE} \approx 200 \mathrm{~cm}^{2} / \mathrm{C}$ ) color more efficiently than the platelets (intrinsic $\mathrm{CE} \approx 150 \mathrm{~cm}^{2} / \mathrm{C}$ ) in $\mathrm{TBA}^{+}$electrolyte.

Single nanocrystal simulations of LSPR reveal the influence of shape and crystalline anisotropy on electrochromic modulation in nanoplatelets and nanorods. Although films of plasmonic nanocrystals have different optical responses than dilute dispersions, due to film reflections and inter-particle LSPR coupling, ${ }^{20,25}$ models of LSPR scattering in single nanocrystal can provide insight on optical modulation. Furthermore, the consistent film thickness and porosity between films of nanorods and nanoplatelets ensure that differences in optical spectra are mostly due to intrinsic material properties. To investigate how anisotropy affects the modulation of LSPR scattering, electromagnetic COMSOL simulations of optical power loss through single particles were modeled for varying degrees of charging (see the Supplemental Information for more detail). The experimentally measured absorption peaks that arise during charging (Figure 2a,b) show very minor shifts in energy despite large changes in intensity. Kim et al. demonstrated that this observation is inconsistent with a model of homogeneous electron accumulation in dispersed h$\mathrm{Cs}: \mathrm{WO}_{3}$ nanocrystals of comparable sizes and shapes, and instead modeled charging (in that case, electron accumulation by a chemical redox reaction) using a model in which an electron-dense core grows while a depleted shell shrinks. ${ }^{24}$ By the same reasoning, we used a growing core/shrinking shell model of charging to simulate electrochromic charging in nanorods and nanoplatelets with morphologies based on the experimental samples. It should be mentioned that these simulations do not capture changes in crystalline dielectric parameters upon ion insertion (e.g. with $\mathrm{Li}^{+}$or $\mathrm{Na}^{+}$), so comparisons with experiment are most directly relevant to charging with non-inserting $\mathrm{TBA}^{+}$electrolytes (Figure 2).

The effects of shape anisotropy were first isolated by simulating LSPR modulation in nanoplatelets and nanorods with crystalline isotropy. In these simulations, the effective mass of the high-mobility c-axis $\left(\mathrm{m}_{\mathrm{e}}{ }^{*}=0.75\right)$ was artificially used for the $\mathrm{a}$ - and b-axes as well (Figure 3a, b). The nanorods have a higher aspect ratio $(A R=1.74)$ than the nanoplatelets $(A R=0.71)$, so peak splitting between a higher energy transverse (x-y) mode and lower energy longitudinal (z) mode is only observed in the nanorods, consistent with classical metal nanoparticle LSPR absorption behavior. ${ }^{17}$ This trend is opposite from the experimental spectra of dispersed nanorods and nanoplatelets (Figure 1c), confirming the counteracting influence of crystalline anisotropy. CE in the simulated spectra is calculated by absorption change at the peak energy per carrier density increase. The intrinsic CE of the nanorod model at the LSPR peak, with an isotropic effective mass $\left(0.45\right.$ per $\left.10^{20} \mathrm{~cm}^{-3}\right)$, is significantly larger than for the nanoplatelet $\left(0.28\right.$ per $\left.10^{20} \mathrm{~cm}^{-3}\right)$. Thus, shape anisotropy alone can influence CE in electrochromic plasmonic nanocrystals. 
a

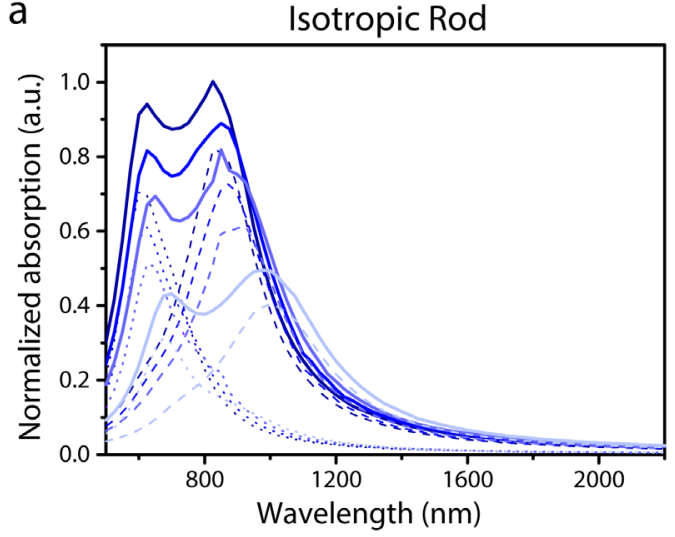

C

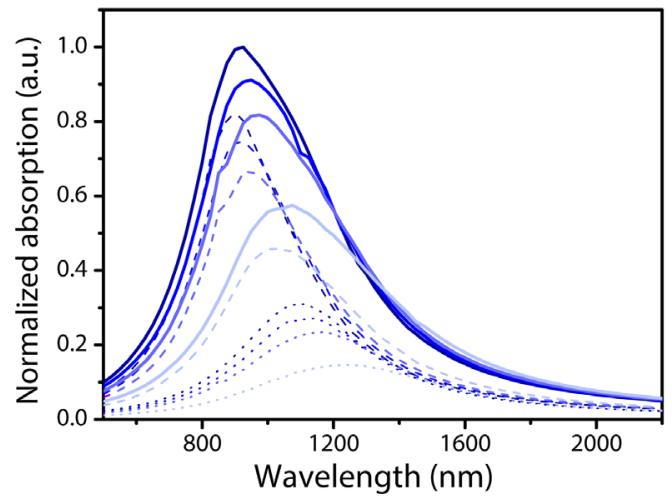

b

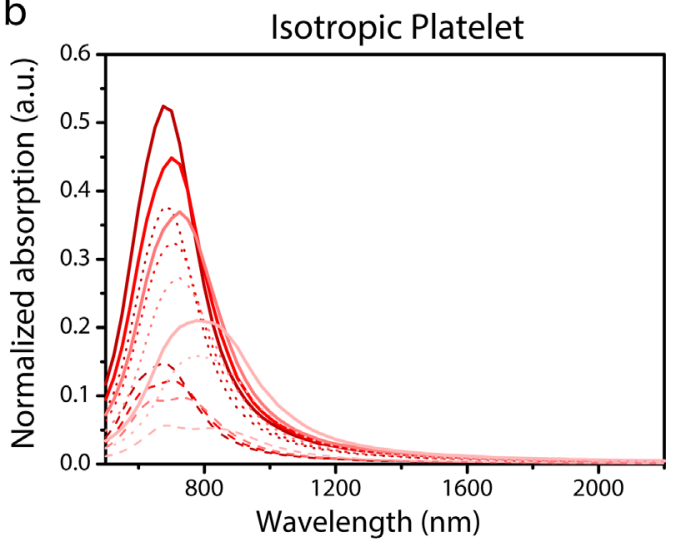

d

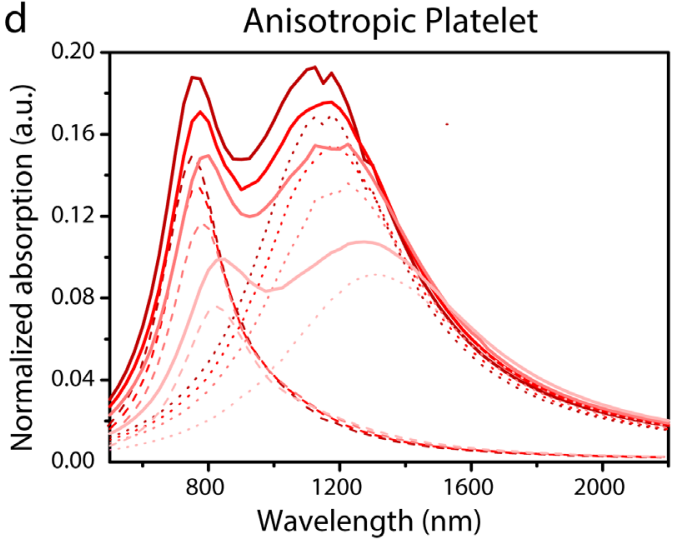

Figure 3. Simulated modulation spectra of nanorods and nanoplatelets. Normalized absorption spectra of crystalline isotropic (using $m_{e}{ }^{*}=0.75$ ) rod (a) and platelet (b), and crystalline anisotropic rod (c) and platelet (d) under the same carrier density changes. Each incremental graph corresponds to the carrier concentration $\left(\mathrm{n}_{\mathrm{e}}\right)$ increase from 1.54, 2.19, 2.44, and $2.65 \times 10^{20} \mathrm{~cm}^{-3}$ sequentially. Dashed lines indicate longitudinal polarization ( $\mathrm{z}$ direction) contributions, and dotted lines indicate transverse polarization ( $\mathrm{x}$ and y direction) contributions. Each isotropic and anisotropic platelet spectrum was normalized with respect to corresponding maximum peak of the rod spectrum.

Only when we include influence of crystalline anisotropy do simulated LSPR spectra resemble the experimental spectra of dispersed nanoplatelets and nanorods. Physically reasonable anisotropic effective mass values (Figure 2c) were used to obtain simulated spectra (Figure 3c,d) upon variable charge accumulation. LSPR peak splitting was observed in the platelets, whereas the transverse and longitudinal LSPR modes overlap in the rods, giving the appearance of a single peak. This result is consistent with experimental spectra of dispersed nanorods and nanoplatelets (Figure 1c), and confirms earlier simulations of LSPR in anisotropic h-Cs: $\mathrm{WO}_{3}$ nanocrystals. ${ }^{24}$ Similar trends in peak splitting are observed in the experimental spectra of electrochromic films of platelets and rods (Figure 2a,b), although LSPR coupling broadens and red-shifts the LSPR peaks. The influence of crystalline anisotropy on peak splitting can be explained by the Drude theory of LSPR. The LSPR peak frequency $\left(\omega_{L S P R}\right)$ depends on the bulk plasma frequency $\left(\omega_{p}=\sqrt{\frac{n e^{2}}{m_{e}^{*} \varepsilon_{0}}}\right.$ ), where $e$ is electron charge, $m_{e}{ }^{*}$ is electron effective mass, and $n$ is free electron density. The large effective mass $\left(\mathrm{m}_{\mathrm{e}}{ }^{*}=1.61\right)$ along $\mathrm{x}$ and $\mathrm{y}$ crystalline axes red-shifts and diminishes the transverse LSPR mode ( $\mathrm{x}$ and y polarization) for both rods and platelets, compared to the isotropic case 
(Figure 3). Thus, both LSPR modes overlap in the high aspect-ratio rods, whereas the LSPR peaks split in energy for the platelets.

In addition to the peak position, crystalline anisotropy also impacts modulation in LSPR intensity. Simulations that include crystalline anisotropy reveal that LSPR peak intensity increases much more for rods than for platelets upon the same accumulation of charge (Figure 3c,d). Intrinsic CE is an order of magnitude higher in nanorod $\left(0.38\right.$ per $\left.10^{20} \mathrm{~cm}^{-3}\right)$ than in nanoplatelet $\left(0.08\right.$ per $\left.10^{20} \mathrm{~cm}^{-3}\right)$. This difference can be explained by the combined effects of shape anisotropy and anisotropic electron effective mass. The longitudinal LSPR mode in nanorods has a significantly higher intrinsic CE, and maximum modulation, even in the absence of crystalline anisotropy (Figure 3a,c). The large effective mass along the $x-y$ direction in actual nanocrystals $\left(\mathrm{m}_{\mathrm{e}}{ }^{*}=1.61\right)$ magnifies this difference. LSPR intensity scales with the plasma frequency, $\omega_{p}$, so a large effective mass along the polarization direction results in weaker interaction with light. ${ }^{25}$ The high- $\mathrm{m}_{\mathrm{e}}{ }^{*}$ transverse LSPR mode has much lower intensity and intrinsic CE, than the low- $\mathrm{m}_{\mathrm{e}}{ }^{*}$ longitudinal mode in both shapes because of crystalline anisotropy. Since the longitudinal mode is relatively less intense in platelets due to shape anisotropy, the total modulation in LSPR of platelets, including contributions from both diminished transverse and weak longitudinal modes, is therefore much less intense than in rods. Electrochromic films of nanorods do indeed show higher intrinsic CE than nanoplatelets (Figure 2d), although the difference is less dramatic than suggested by single nanocrystal simulations. LSPR coupling ${ }^{20,25}$ and polydispersity ${ }^{24}$ may account for this weaker-than-expected sensitivity of intrinsic CE to nanocrystal shape. Overall, the combination of both sources of anisotropy influence both intrinsic $\mathrm{CE}$ and total optical modulation, with practical consequences for near-infrared electrochromic devices.

Optical modulation in h-Cs: $\mathrm{WO}_{3}$ nanocrystals increases with accumulated charge, so intrinsic charge capacity influences the maximum optical modulation a film can achieve. In an electrochromic film, this accumulated charge is controlled by the applied voltage, but properties of the electrode and electrolyte can change how much charge is stored at a particular voltage. Unlike $\mathrm{TBA}^{+}$cations, which are too bulky to intercalate into h-Cs: $\mathrm{WO}_{3}$ nanocrystals, ${ }^{36} \mathrm{Na}^{+}$and $\mathrm{Li}^{+}$are both small enough to diffuse through interstitial voids. Charge capacity depends on which interstitial voids can be occupied by each cation. $\mathrm{Na}^{+}$can access hexagonal tunnel sites in the hexagonal $\mathrm{WO}_{3}$ lattice and $\mathrm{Li}^{+}$is small enough to also occupy trigonal cavities as well as hexagonal tunnel sites, thus providing larger charge capacity. ${ }^{26,37-38}$ Electrochromic nanorod and nanoplatelet films reflect these trends; the intrinsic capacity is smallest with bulky, non-inserting $\mathrm{TBA}^{+}$and largest with small $\mathrm{Li}^{+}$electrolyte (Table 2). The maximum optical modulation that can be achieved in each film correlates with charge capacity, with the smallest total optical modulation in $\mathrm{TBA}^{+}$electrolyte, intermediate modulation with $\mathrm{Na}^{+}$, and the greatest modulation using $\mathrm{Li}^{+}$(Table 2 and Figures $2 \mathrm{~d}, \mathbf{4 b}, \mathbf{S 6}$ ).

The shape of the nanocrystals influences the intrinsic charge capacity with $\mathrm{Na}^{+}$electrolytes because the $\mathrm{Cs}^{+}$incorporated in the crystalline structure acts as a barrier to anisotropic charge diffusion. Shape has only minor impacts on intrinsic capacity with either $\mathrm{TBA}^{+}$cations, which are too bulky to insert, or $\mathrm{Li}^{+}$, which is small enough to easily diffuse throughout the lattice. However, in $\mathrm{Na}^{+}$electrolytes a clear difference is observed. Nanoplatelets have significantly higher intrinsic charge capacity than nanorods when charged using $\mathrm{Na}^{+}$(Table 2). $\mathrm{Na}^{+}$is known to diffuse only through one-dimensional hexagonal tunnel sites $\left(\right.$ Figure 1f). ${ }^{26}$ Given the size of $\mathrm{Cs}^{+}$(radius $=167 \mathrm{pm}$ ) and $\mathrm{Na}^{+}$(radius $=102 \mathrm{pm}$ ) compared to the size of the hexagonal tunnel site (end-to-end distance $=501 \mathrm{pm}$ ), the existence of $\mathrm{Cs}^{+}$in the hexagonal tunnel site is expected to hinder the diffusion of $\mathrm{Na}^{+} \cdot{ }^{39} \mathrm{We}$ surmise $\mathrm{Li}^{+}$can probably access hexagonal tunnel sites, including sites blocked by $\mathrm{Cs}^{+}$, because of its smaller size (radius $=76 \mathrm{pm}$ ). A similar blocking phenomenon 
is observed in the battery material $\mathrm{LiMnBO}_{3}$. Larger particles of $\mathrm{LiMnBO}_{3}$ have reduced intrinsic capacity because $\mathrm{Mn}_{\mathrm{Li}}$ antisite defects limit the one-dimensional diffusion of $\mathrm{Li}^{+}$into the lattice. ${ }^{40}$ Figure $4 \mathbf{a}$ illustrates how these 1D diffusion barriers impact charge capacity in platelets and rods. Most of the nanoplatelet volume is accessible to $\mathrm{Na}^{+}$from accessible hexagonal tunnels near the surface, but only a smaller fraction of these sites can be accessed in nanorods due to $\mathrm{Cs}^{+}$blocking 1D tunnel diffusion. Even though ICP analysis (performed by the Galbraith Laboratories, TN) indicates that the actual Cs: W ratio is lower in nanorods (0.38) than in nanoplatelets $(0.49)$, the shape effect still leads to larger intrinsic capacity in the nanoplatelets. It should be noted that ICP analysis gives a higher $\mathrm{Cs}: \mathrm{W}$ ratio than the theoretical maximum (0.33) of hexagonal tungsten oxide, ${ }^{41}$ which likely originates from Cs bound at or near the nanocrystal surface.

Even though $\mathrm{Na}^{+}$and $\mathrm{Li}^{+}$ions insert into the lattice, intrinsic $\mathrm{CE}$ of nanorods is still higher than nanoplatelets for each electrolyte (Table 2). This persistent difference suggests that ion insertion does not qualitatively change the anisotropy in effective mass and corresponding effects on LSPR energy and intensity in h-Cs: $\mathrm{WO}_{3}$ nanocrystals. Related, theoretical study has demonstrated that $\mathrm{Na}^{+}$insertion does not have a large impact on the band structure of cubic tungsten oxide. ${ }^{42} \mathrm{CE}$ in $\mathrm{Na}^{+}$electrolyte is similar to that in $\mathrm{TBA}^{+}$electrolyte, but higher than that for $\mathrm{Li}^{+}$electrolyte, and in each case $\mathrm{CE}$ is higher for rods than platelets. $\mathrm{Na}^{+}$insertion sites are optically active hexagonal tunnels, the same sites already occupied by the dopant $\mathrm{Cs}^{+}$. These sites are expected from our earlier work on monoclinic tungsten oxide nanorods to give rise to high $\mathrm{CE} .{ }^{26}$ However, $\mathrm{Li}^{+}$can also occupy optically inactive trigonal cavity sites, leading to lower CE. ${ }^{26,37}$

a

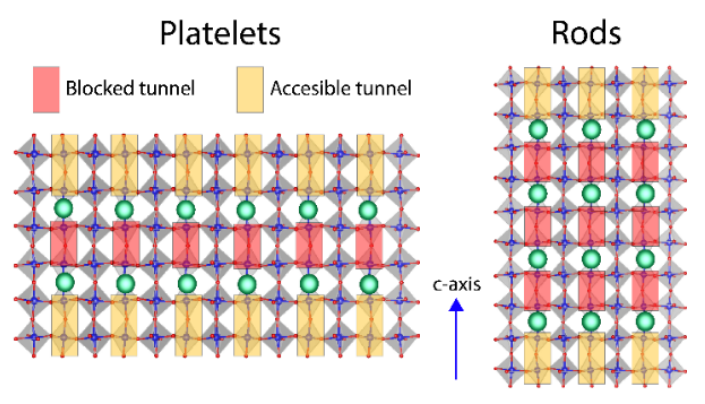

b

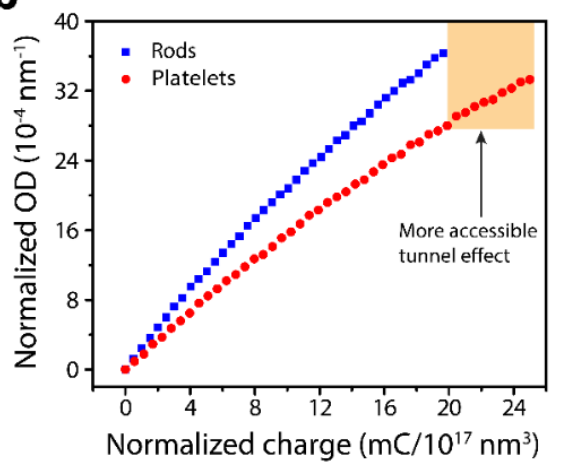

Figure 4. Crystalline and shape anisotropy effect on charge capacity with $\mathrm{Na}^{+}$electrolyte. (a) Graphical representation of accessible $\mathrm{Na}^{+}$insertion sites in platelets and rods assuming similar cesium ratio. (b) Normalized charge and OD at $1700 \mathrm{~nm}$ using $\mathrm{Na}^{+}$electrolyte.

Visible transparency with selective near-infrared modulation is of interest for controlling thermal gain while maintaining daylighting. ${ }^{43-45}$ In our study, significant modulation of transmission over a broad range of near-infrared wavelengths is achieved while maintaining high visible transparency. Figure S7 shows the modulation achieved by thin (thickness $\approx 130 \mathrm{~nm}$ ) electrochromic films of nanorods and nanoplatelets in $\mathrm{Na}^{+}$electrolyte. The large intrinsic near-infrared modulation achieved in these materials would allow for very thin electrochromic coatings without much sacrifice in visible transparency. These materials may be promising alternatives to ITO nanocrystal coatings, which modulate most effectively at longer wavelengths, less well-matched to the solar infrared. ${ }^{46}$ 
In summary, we have investigated how shape and crystalline anisotropy affects the intrinsic charge capacity and $\mathrm{CE}$ of h-Cs: $\mathrm{WO}_{3}$ nanocrystals in electrochromic films. We demonstrate a means to measure these intrinsic properties through careful control of particle morphology and film properties. First, two nanocrystal samples with different aspect ratio in shape were synthesized with near-identical phase, volume, and surface area. Next, uniform thin films of each nanocrystal shape were fabricated with controlled porosity, thickness, and projected area. Finally, we obtained intrinsic material properties of electrochromic nanocrystals from in situ spectroelectrochemical measurements of films, and compared these values for different electrolytes. Both crystalline and shape anisotropy were observed to affect intrinsic $\mathrm{CE}$ and charge capacity during electrochromic modulation. The selection of electrolyte cation had a strong influence on intrinsic charge capacity, and thus total optical modulation. Small cations such as $\mathrm{Li}^{+}$and $\mathrm{Na}^{+}$provide higher charge capacity because they can insert into the lattice. However, nanocrystal shape influenced charge capacity in $\mathrm{Na}^{+}$electrolyte because of the anisotropic blocking of $\mathrm{Na}^{+}$diffusion by $\mathrm{Cs}^{+}$in onedimensional diffusion channels. Our study supports the importance of crystalline and shape anisotropy for the practical development of metal oxide semiconductor electrochromic devices.

\section{ASSOCIATED CONTENT}

Supporting Information is available free of charge on the ACS Publication website at DOI: 10.1021/acsenergylett.XXXXXXX. Experimental, characterization, and modeling details, nanocrystal size distribution, SEM, optical modulation, transmittance, and ellipsometric porosimetry data.

\section{AUTHOR INFORMATION}

ORCID: Delia J. Milliron: 0000-0002-8737-451X

Sungyeon Heo: 0000-0001-8690-5748

Shin Hum Cho: 0000-0002-0271-116X

Clayton J. Dahlman: 0000-0002-4555-4846

Ankit Agrawal: 0000-0001-7311-7873

Notes: The authors declare the following competing financial interests: D.J.M. has a financial interest in Heliotrope Technologies, a company pursuing commercial development of electrochromic devices.

\section{ACKNOWLEDGEMENTS}

S.H. and D.J.M acknowledge funding from Companhia Brasileira de Metallurgia e Mineração (CBMM, UTA16-000941) and the Welch Foundation (F-1848). Support was also provided by the National Science Foundation, including CHE-1905263 and CBET-1704634, and the Fulbright Program (IIE-15151071). XRD was performed at Texas Materials Institute, UT Austin. S.H. thanks Dr. Jongwook Kim for helpful conversation of ICP measurement. 


\section{REFERENCES}

(1) Runnerstrom, E. L.; Llordés, A.; Lounis, S. D.; Milliron, D. J. Nanostructured Electrochromic Smart Windows: Traditional Materials and NIR-Selective Plasmonic Nanocrystals. Chem. Commun. 2014, 50, 10555-10572.

(2) Agrawal, A.; Cho, S. H.; Zandi, O.; Ghosh, S.; Johns, R. W.; Milliron, D. J. Localized Surface Plasmon Resonance in Semiconductor Nanocrystals. Chem. Rev. 2018, 118, 3121-3207.

(3) Granqvist, C. G.; Arvizu, M. A.; Bayrak Pehlivan, İ; Qu, H.-Y.; Wen, R.-T.; Niklasson, G. A. Electrochromic Materials and Devices for Energy Efficiency and Human Comfort in Buildings: A Critical Review. Electrochim. Acta 2018, 259, 1170-1182.

(4) Llordés, A.; Garcia, G.; Gazquez, J.; Milliron, D. J. Tunable Near-Infrared and Visible-Light Transmittance in Nanocrystal-in-Glass Composites. Nature 2013, 500, 323-326.

(5) Granqvist, C. G. Electrochromics for Smart Windows: Oxide-Based Thin Films and Devices. Thin Solid Films 2014, 564, 1-38.

(6) Novo, C.; Funston, A. M.; Gooding, A. K.; Mulvaney, P. Electrochemical Charging of Single Gold Nanorods. J. Am. Chem. Soc. 2009, 131, 14664-14666.

(7) Heo, S.; Kim, J.; Ong, G. K.; Milliron, D. J. Template-Free Mesoporous Electrochromic Films on Flexible Substrates from Tungsten Oxide Nanorods. Nano Lett. 2017, 17, 5756-5761.

(8) Barawi, M.; De Trizio, L.; Giannuzzi, R.; Veramonti, G.; Manna, L.; Manca, M. Dual Band Electrochromic Devices Based on Nb-Doped $\mathrm{TiO}_{2}$ Nanocrystalline Electrodes. ACS Nano 2017, 11, 35763584.

(9) Dahlman, C. J.; Tan, Y.; Marcus, M. A.; Milliron, D. J. Spectroelectrochemical Signatures of Capacitive Charging and Ion Insertion in Doped Anatase Titania Nanocrystals. J. Am. Chem. Soc. 2015, $137,9160-9166$.

(10) Lu, H.-C.; Ghosh, S.; Katyal, N.; Henkelman, G.; Lankanpal, V. S.; Milliron, D. Synthesis and Dual-Mode Electrochromism of Anisotropic Monoclinic $\mathrm{Nb}_{12} \mathrm{O}_{29}$ Colloidal Nanoplatelets, 2020. ChemRxiv. Preprint. https://doi.org/10.26434/chemrxiv.12108612.v1

(11) Zhang, S.; Li, Y.; Zhang, T.; Cao, S.; Yao, Q.; Lin, H.; Ye, H.; Fisher, A.; Lee, J. Y. Dual-Band Electrochromic Devices with a Transparent Conductive Capacitive Charge-Balancing Anode. ACS Appl. Mater. Interfaces 2019, 11, 48062-48070.

(12) Cao, S.; Zhang, S.; Zhang, T.; Lee, J. Y. Fluoride-Assisted Synthesis of Plasmonic Colloidal TaDoped $\mathrm{TiO}_{2}$ Nanocrystals for Near-Infrared and Visible-Light Selective Electrochromic Modulation. Chem. Mater. 2018, 30, 4838-4846.

(13) Barawi, M.; Veramonti, G.; Epifani, M.; Giannuzzi, R.; Sibillano, T.; Giannini, C.; Rougier, A.; Manca, M. A Dual Band Electrochromic Device Switchable across Four Distinct Optical Modes. J. Mater. Chem. A 2018, 6, 10201-10205.

(14) Yao, D. D.; Rani, R. A.; O’Mullane, A. P.; Kalantar-zadeh, K.; Ou, J. Z. High Performance Electrochromic Devices Based on Anodized Nanoporous $\mathrm{Nb}_{2} \mathrm{O}_{5}$. J. Phys. Chem. C 2014, 118, 476-481. 
(15) Axmann, P.; Stinner, C.; Wohlfahrt-Mehrens, M.; Mauger, A.; Gendron, F.; Julien, C. M. Nonstoichiometric $\mathrm{LiFePO}_{4}$ : Defects and Related Properties. Chem. Mater. 2009, 21, 1636-1644.

(16) Kuss, C.; Liang, G.; Schougaard, S. B. Atomistic Modeling of Site Exchange Defects in Lithium Iron Phosphate and Iron Phosphate. J. Mater. Chem. 2012, 22, 24889-24893.

(17) Kelly, K. L.; Coronado, E.; Zhao, L. L.; Schatz, G. C. The Optical Properties of Metal Nanoparticles: The Influence of Size, Shape, and Dielectric Environment. J. Phys. Chem. B 2003, 107, 668-677.

(18) Perezjuste, J.; Pastorizasantos, I.; Lizmarzan, L.; Mulvaney, P. Gold Nanorods: Synthesis, Characterization and Applications. Coord. Chem. Rev. 2005, 249, 1870-1901.

(19) Cho, S. H.; Ghosh, S.; Berkson, Z. J.; Hachtel, J. A.; Shi, J.; Zhao, X.; Reimnitz, L. C.; Dahlman, C. J.; Ho, Y.; Yang, A.; et al. Syntheses of Colloidal F: $\operatorname{In}_{2} \mathrm{O}_{3}$ Cubes: Fluorine-Induced Faceting and Infrared Plasmonic Response. Chem. Mater. 2019, 31, 2661-2676.

(20) Tandon, B.; Agrawal, A.; Heo, S.; Milliron, D. J. Competition between Depletion Effects and Coupling in the Plasmon Modulation of Doped Metal Oxide Nanocrystals. Nano Lett. 2019, 19, 2012-2019.

(21) Zandi, O.; Agrawal, A.; Shearer, A. B.; Reimnitz, L. C.; Dahlman, C. J.; Staller, C. M.; Milliron, D. J. Impacts of Surface Depletion on the Plasmonic Properties of Doped Semiconductor Nanocrystals. Nat. Mater. 2018, 17, 710-717.

(22) Gibbs, S. L.; Staller, C. M.; Milliron, D. J. Surface Depletion Layers in Plasmonic Metal Oxide Nanocrystals. Acc. Chem. Res. 2019, 52, 2516-2524.

(23) Pei, Y.; LaLonde, A. D.; Wang, H.; Snyder, G. J. Low Effective Mass Leading to High Thermoelectric Performance. Energy Environ. Sci. 2012, 5, 7963-7969.

(24) Kim, J.; Agrawal, A.; Krieg, F.; Bergerud, A.; Milliron, D. J. The Interplay of Shape and Crystalline Anisotropies in Plasmonic Semiconductor Nanocrystals. Nano Lett. 2016, 16, 3879-3884.

(25) Dahlman, C. J.; Agrawal, A.; Staller, C. M.; Adair, J.; Milliron, D. J. Anisotropic Origins of Localized Surface Plasmon Resonance in n-Type Anatase $\mathrm{TiO}_{2}$ Nanocrystals. Chem. Mater. 2019, 31, 502511.

(26) Heo, S.; Dahlman, C. J.; Staller, C. M.; Jiang, T.; Dolocan, A.; Korgel, B. A.; Milliron, D. J. Enhanced Coloration Efficiency of Electrochromic Tungsten Oxide Nanorods by Site Selective Occupation of Sodium Ions. Nano Lett. 2020, 20, 2072-2079.

(27) Giannuzzi, R.; Scarfiello, R.; Sibillano, T.; Nobile, C.; Grillo, V.; Giannini, C.; Cozzoli, P. D.; Manca, M. From Capacitance-Controlled to Diffusion-Controlled Electrochromism in One-Dimensional Shape-Tailored Tungsten Oxide Nanocrystals. Nano Energy 2017, 41, 634-645.

(28) Prinz, H.; Müller, U.; Ha-Eierdanz, M.-L. Ein Neues Syntheseverfahren Für Die Wolframbronze $\mathrm{Cs}_{0,29} \mathrm{WO}_{3}$. Z. Anorg. Allg. Chem. 1992, 609, 95-98.

(29) Yang, P.; Sun, P.; Chai, Z.; Huang, L.; Cai, X.; Tan, S.; Song, J.; Mai, W. Large-Scale Fabrication of Pseudocapacitive Glass Windows That Combine Electrochromism and Energy Storage. Angew. Chem. Int. Ed. 2014, 53, 11935-11939. 


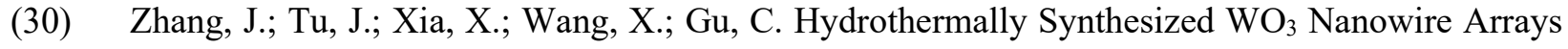
with Highly Improved Electrochromic Performance. J. Mater. Chem. 2011, 21, 5492-5498.

(31) Gaupp, C. L.; Welsh, D. M.; Rauh, R. D.; Reynolds, J. R. Composite Coloration Efficiency Measurements of Electrochromic Polymers Based on 3,4-Alkylenedioxythiophenes. Chem. Mater. 2002, 14, 3964-3970.

(32) Chen, Y.; Bi, Z.; Li, X.; Xu, X.; Zhang, S.; Hu, X. High-Coloration Efficiency Electrochromic Device Based on Novel Porous $\mathrm{TiO}_{2} @$ Prussian Blue Core-Shell Nanostructures. Electrochim. Acta 2017, $224,534-540$.

(33) Zhan, Y.; Tan, M. R. J.; Cheng, X.; Tan, W. M. A.; Cai, G. F.; Chen, J. W.; Kumar, V.; Magdassi, S.; Lee, P. S. Ti-Doped $\mathrm{WO}_{3}$ Synthesized by a Facile Wet Bath Method for Improved Electrochromism. $J$. Mater. Chem. C 2017, 5, 9995-10000.

(34) Jain, P. K.; Lee, K. S.; El-Sayed, I. H.; El-Sayed, M. A. Calculated Absorption and Scattering Properties of Gold Nanoparticles of Different Size, Shape, and Composition: Applications in Biological Imaging and Biomedicine. J. Phys. Chem. B 2006, 110, 7238-7248.

(35) Staller, C. M.; Gibbs, S. L.; Saez Cabezas, C. A.; Milliron, D. J. Quantitative Analysis of Extinction Coefficients of Tin-Doped Indium Oxide Nanocrystal Ensembles. Nano Lett. 2019, 19, 8149-8154.

(36) Kim, J.; Ong, G. K.; Wang, Y.; LeBlanc, G.; Williams, T. E.; Mattox, T. M.; Helms, B. A.; Milliron, D. J. Nanocomposite Architecture for Rapid, Spectrally-Selective Electrochromic Modulation of Solar Transmittance. Nano Lett. 2015, 15, 5574-5579.

(37) Balaji, S.; Djaoued, Y.; Albert, A.-S.; Ferguson, R. Z.; Brüning, R. Hexagonal Tungsten Oxide Based Electrochromic Devices: Spectroscopic Evidence for the Li Ion Occupancy of Four-Coordinated Square Windows. Chem. Mater. 2009, 21, 1381-1389.

(38) Evans, R. C.; Ellingworth, A.; Cashen, C. J.; Weinberger, C. R.; Sambur, J. B. Influence of SingleNanoparticle Electrochromic Dynamics on the Durability and Speed of Smart Windows. Proc. Natl. Acad. Sci. U.S.A. 2019, 116, 12666-12671.

(39) Zhai, D.; Li, B.; Xu, C.; Du, H.; He, Y.; Wei, C.; Kang, F. A Study on Charge Storage Mechanism of $\alpha-\mathrm{MnO}_{2}$ by Occupying Tunnels with Metal Cations $\left(\mathrm{Ba}^{2+}, \mathrm{K}^{+}\right)$. J. Power Sources 2011, 196, 7860-7867.

(40) Kim, J. C.; Seo, D.-H.; Chen, H.; Ceder, G. The Effect of Antisite Disorder and Particle Size on Li Intercalation Kinetics in Monoclinic $\mathrm{LiMnBO}_{3}$. Adv. Energy Mater. 2015, 5, 1401916.

(41) Lee, Y.; Lee, T.; Jang, W.; Soon, A. Unraveling the Intercalation Chemistry of Hexagonal Tungsten Bronze and Its Optical Responses. Chem. Mater. 2016, 28, 4528-4535.

(42) Ingham, B.; Hendy, S. C.; Chong, S. V.; Tallon, J. L. Density-Functional Studies of Tungsten Trioxide, Tungsten Bronzes, and Related Systems. Phys. Rev. B 2005, 72, 075109.

(43) Chaudhary, A.; Pathak, D. K.; Tanwar, M.; Koch, J.; Pfnür, H.; Kumar, R. PolythiophenenanoWO $\mathrm{W}_{3}$ Bilayer as an Electrochromic Infrared Filter: A Transparent Heat Shield. J. Mater. Chem. C 2020, $8,1773-1780$.

(44) Khandelwal, H.; Schenning, A. P. H. J.; Debije, M. G. Infrared Regulating Smart Window Based on Organic Materials. Adv. Energy Mater. 2017, 7, 1602209. 
(45) Nguyen, T. D.; Yeo, L. P.; Kei, T. C.; Mandler, D.; Magdassi, S.; Tok, A. I. Y. Efficient Near Infrared Modulation with High Visible Transparency Using $\mathrm{SnO}_{2}-\mathrm{WO}_{3}$ Nanostructure for Advanced Smart Windows. Adv. Optical Mater. 2019, 7, 1801389.

(46) Garcia, G.; Buonsanti, R.; Runnerstrom, E. L.; Mendelsberg, R. J.; Llordes, A.; Anders, A.; Richardson, T. J.; Milliron, D. J. Dynamically Modulating the Surface Plasmon Resonance of Doped Semiconductor Nanocrystals. Nano Lett. 2011, 11, 4415-4420. 